\title{
Tratamento com Sucesso de um Caso de Tromboembolismo Pulmonar Maciço com Uso de Cateter de Aspiração a Vácuo
}

\author{
André Eduardo Gomes ${ }^{1,2}$, Eduardo Arantes Nogueira ${ }^{1,2}$, Fábio Coelho Barroso ${ }^{1}$
}

\section{RESUMO}

Relatamos um caso de tromboembolismo pulmonar maciço em paciente idosa, com grande instabilidade clínica e hemodinâmica, que foi submetida a intervenção percutânea precoce. Realizamos embolectomia com cateter de aspiração Pronto 0,035" 10 F e trombólise intra-arterial (dose reduzida), com excelente resultado.

DESCRITORES: Tromboembolismo pulmonar. Embolectomia. Sucção/métodos. Cateterismo.

0 tromboembolismo pulmonar é uma situação clínica grave e com alta mortalidade. Por ser pouco diagnosticado, sua incidência real não é conhecida. Estima-se que ocorram mais de $600 \mathrm{mil}$ casos por ano e é a terceira causa de doença cardiovascular nos Estados Unidos ${ }^{1,2}$, causando de 150 mil a 200 mil mortes por ano $^{3}$. Nos pacientes com tromboembolismo pulmonar maciço, falência do ventrículo direito e instabilidade hemodinâmica, a mortalidade é elevada, variando de $25 \%$ a $65 \%{ }^{4}$. A intervenção rápida e o restabelecimento do fluxo pulmonar diminuem a mortalidade e melhoram a qualidade de vida ${ }^{5}$.

\section{RELATO DO CASO}

Paciente do sexo feminino, 94 anos, deu entrada no pronto atendimento de nossa Instituição (Fundação Centro Médico de Campinas - Campinas, SP) no dia 30 de setembro de 2008 com quadro agudo de taquidispneia, cianose generalizada e hipotensão arterial grave (pressão arterial $82 / 40 \mathrm{mmHg}$ ). Segundo relato dos fa-

\footnotetext{
Fundação Centro Médico de Campinas - Campinas, SP, Brasil.

2 Universidade Estadual de Campinas (Unicamp) - Campinas, SP, Brasil.

Correspondência: André Eduardo Gomes. Rua Maria Monteiro, 1286, apto 51 - Cambuí - Campinas, SP, Brasil - CEP 13025-152.

E-mail: aegomes@cardiol.br

Recebido em: 16/4/2009 • Aceito em: 26/8/2009
}

\begin{abstract}
Clinical Case: Massive Pulmonary Embolism

Successfully Treated with Aspiration Catheter

We report here the use of the Pronto .035" $10 \mathrm{~F}$ aspiration catheter and intra-arterial trombolysis (reduced dose) in the successful treatment of an elderly patient with a massive acute pulmonary thromboembolism and hemodynamic collapse submitted to early percutaneous intervention.
\end{abstract}

DESCRIPTORS: Pulmonary embolism. Embolectomy. Suction/ methods. Catheterization.

miliares, a paciente apresentou dispneia súbita, perda da consciência e um episódio de crise convulsiva. Como antecedente, havia sido submetida a cirurgia para implante de prótese parcial de quadril esquerdo há 26 dias. Em razão do quadro de insuficiência respiratória aguda e choque, foram realizadas intubação orotraqueal e infusão de dopamina e a paciente foi encaminhada à Unidade de Terapia Intensiva (UTI). A hipótese primária foi de tromboembolismo pulmonar, pelo histórico de pós-operatório tardio de cirurgia ortopédica e ausência de correta profilaxia para tromboembolismo venoso. O eletrocardiograma apresentava taquicardia sinusal e bloqueio de ramo direito de $1^{\circ}$ grau. A radiografia de tórax demonstrou somente leve aumento da área cardíaca (átrio e ventrículo esquerdos). Os resultados dos exames laboratoriais foram: hemoglobina = $13 \mathrm{~g} / \mathrm{dl}$; plaquetas $=274 \mathrm{mil} / \mathrm{mm}^{3}$; tempo de protrombina $=16,1 \mathrm{~s} ; \mathrm{RNI}=1,56$; creatinina $=1,38 \mathrm{mg} / \mathrm{dl}$; e troponina $=1,5 \mathrm{ng} / \mathrm{ml}(\mathrm{nl}<1)$. A gasometria arterial demonstrava: $\mathrm{pH}=7,44 ; \mathrm{pO}_{2}=53,7 \mathrm{mmHg} ; \mathrm{pCO}_{2}=$ $28,1 \mathrm{mmHg} ; \mathrm{HCO}_{3}=18,9 \mathrm{mmol} / \mathrm{l}$; e saturação de $\mathrm{O}_{2}=$ $85,1 \%$. Em decorrência da grande suspeita de tromboembolismo pulmonar, inicialmente foi realizado ecocardiograma transtorácico, que evidenciou aumento do átrio esquerdo (46 mm) e insuficiência tricúspide moderada com hipertensão pulmonar (pressão sistólica de $45 \mathrm{mmHg}$ ). A angiotomografia de tórax evidenciou imagens de trombos promovendo uma oclusão vascular parcial na origem das artérias pulmonares (configuração em "V') e nas artérias lobares direitas. Após a 
Gomes AE, et al. Tratamento com Sucesso de um Caso de Tromboembolismo Pulmonar Maciço com Uso de Cateter de Aspiração a Vácuo. Rev Bras Cardiol Invas. 2009;17(3):414-7.

confirmação de tromboembolismo pulmonar maciço, a paciente apresentou choque cardiogênico refratário a drogas vasoativas (dopamina e noradrenalina). A trombólise sistêmica foi contraindicada pelo alto risco de sangramentos e optou-se por levar a paciente à sala de hemodinâmica para tentativa de embolectomia percutânea.

Depois de realizada punção de artéria femoral direita e da colocação de introdutor $5 \mathrm{~F}$, foi feito registro da pressão arterial $(94 / 56 \mathrm{mmHg}$ ) associado a punção de veia femoral direita e colocação de introdutor 7 F. Com um cateter pig tail 5 F verificou-se a pressão sistólica de artéria pulmonar $(58 \mathrm{mmHg})$ e, a seguir, foram realizadas arteriografia do tronco da artéria pulmonar e arteriografias seletivas de pulmonares direita e esquerda, com visualização de grande quantidade de trombos, bilateralmente, sendo o maior localizado na porção distal da artéria pulmonar direita (Figura 1), comprometendo quase todo o diâmetro da artéria e confirmando o tromboembolismo pulmonar maciço. A seguir, um fio-guia 0,035', curvo, de $260 \mathrm{~cm}$, foi posicionado na artéria pulmonar direita, sendo retirado o introdutor e introduzido o cateter Pronto 0,035" 10 F (Vascular Solutions, Inc., Minneapolis, Estados Unidos), e realizadas várias aspirações a vácuo com saída de grande quantidade de trombos (Figura 2). O procedimento foi também realizado na artéria pulmonar esquerda, com saída de boa quantidade de trombos. Em decorrência da grande quantidade de fragmentos visualizada em ramos pulmonares distais, optou-se pela administração de trombolítico (Actilyse ${ }^{T M} 20 \mathrm{mg}$ ) com o cateter pig tail mantido na artéria pulmonar direita. A arteriografia pulmonar direita, realizada no final do procedimento, demonstrou desobstrução quase

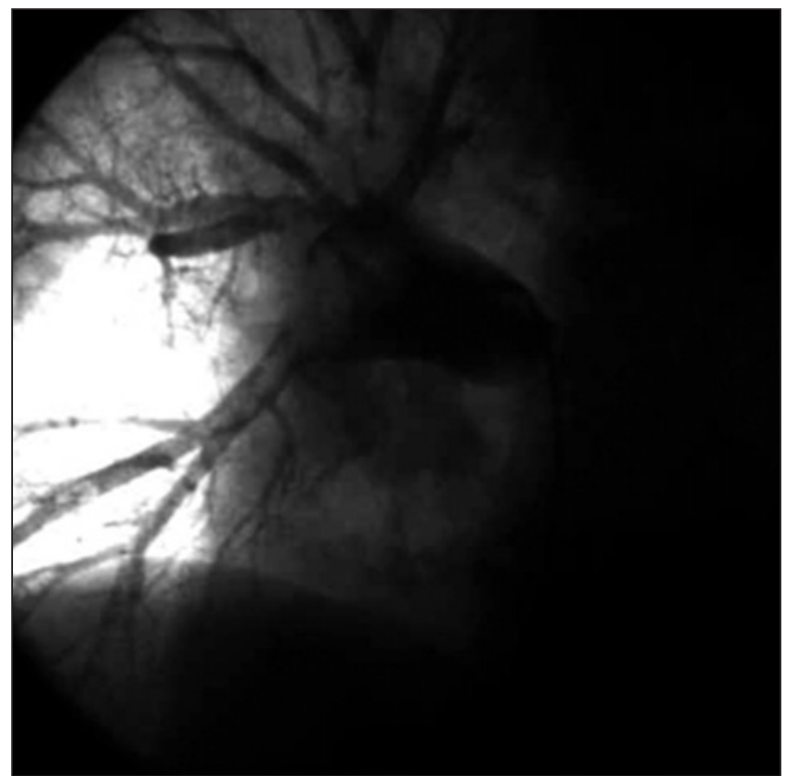

Figura 1 - Arteriografia pulmonar seletiva: grande trombo ocluindo porção distal da artéria pulmonar direita. completa do ramo interlobar e persistência de pequenos fragmentos do trombo, ocluindo parcialmente o ramo lobar superior e ramos segmentares dos lobos médio e inferior (Figura 3).

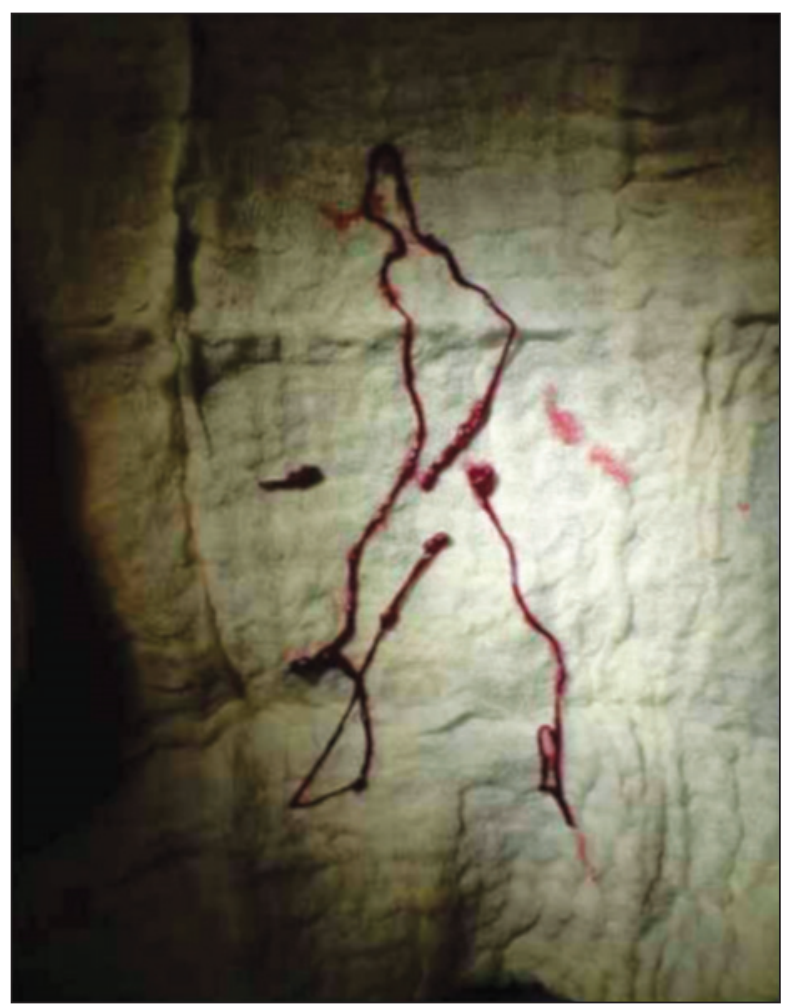

Figura 2 - Grande quantidade de trombos aspirados no procedimento.

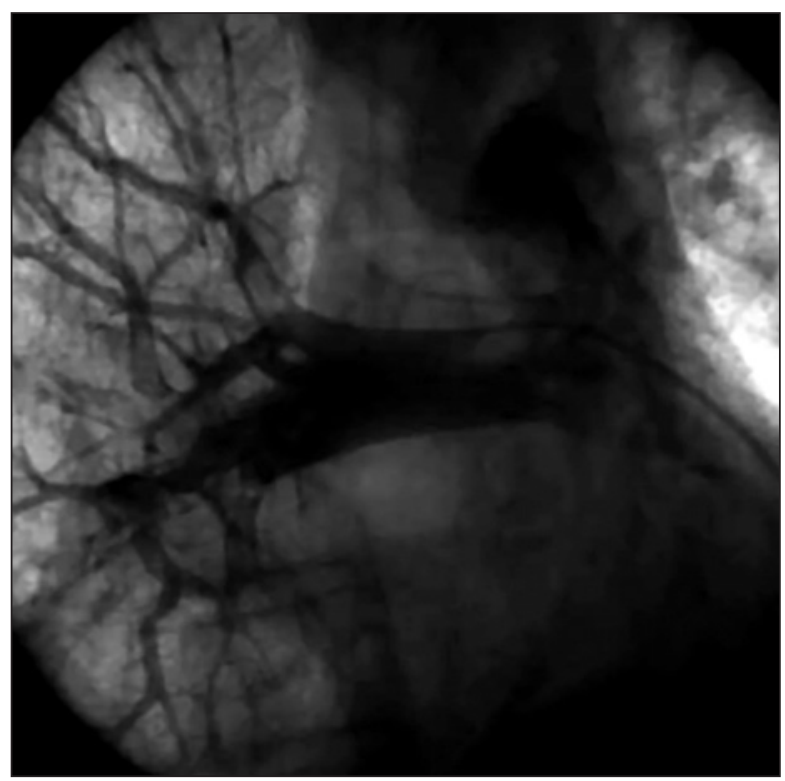

Figura 3 - Arteriografia pulmonar após aspiração: desobstrução quase completa do ramo interlobar e persistência de pequenos fragmentos ocluindo parcialmente o ramo lobar superior e ramos segmentares dos lobos médio e inferior da artéria pulmonar direita. 
A paciente apresentou melhora hemodinâmica, com aumento da pressão arterial $(124 / 80 \mathrm{mmHg})$, e posterior retorno à UTI. Apresentou boa evolução, com suspensão de drogas vasoativas e ventilação mecânica após 48 horas do procedimento. Recebeu alta da UTI no quinto dia de internação em uso de enoxaparina (dose plena) e vancomicina (para tratamento de pneumonia adquirida) e permaneceu assintomática até a alta hospitalar. Manteve-se em uso de dicumarínico e controle de anticoagulação com RNI 2,8 e encaminhamento ambulatorial.

\section{DISCUSSÃO}

O tromboembolismo pulmonar é uma situação clínica grave e com alta mortalidade. No tromboembolismo pulmonar maciço, em decorrência das obstruções grave e aguda do fluxo arterial pulmonar, ocorre aumento rápido da pressão arterial pulmonar, com dilatação do ventrículo direito, levando a cor pulmonale agudo, que rapidamente evolui para diminuição do débito cardíaco e colapso cardiovascular, acarretando rapidamente a morte do paciente $^{6}$. Em caso de tromboembolismo pulmonar maciço com falência do ventrículo direito aguda e instabilidade hemodinâmica, a mortalidade é elevada, variando de 25\% a $65 \%{ }^{4}$. O diagnóstico de tromboembolismo pulmonar maciço é confirmado quando temos na angiografia imagem de trombos na artéria pulmonar principal ou em um ou mais ramos lobares, e um ou mais critérios de falência do ventrículo direito ${ }^{3}$. O diagnóstico rápido e o restabelecimento do fluxo pulmonar com melhora hemodinâmica diminui a mortalidade e melhora a qualidade de vida ${ }^{5}$. O tratamento clássico, com anticoagulação, trombólise sistêmica e trombectomia cirúrgica, não conseguiu reduzir a mortalidade no tromboembolismo pulmonar maciço. A trombólise sistêmica tem sido a terapêutica padrão indicada no tromboembolismo pulmonar com grave instabilidade hemodinâmica. Entretanto, evidências recentes não têm confirmado o benefício da trombólise: similar mortalidade foi encontrada nos pacientes com tromboembolismo pulmonar tratados com heparinização vs. trombólise sistêmica $(46,3 \% \text { vs. } 55,1 \% ; P=0,44)^{7}$. Além disso, $8 \%$ apresentam falha com o tratamento e 33\% têm contraindicação absoluta para a trombólise sistêmica ${ }^{8,9}$. A embolectomia cirúrgica envolve esternotomia e número limitado de pacientes e associa-se a grandes complicações e alta mortalidade $(46 \%)^{10}$. Nos últimos anos, com o desenvolvimento de técnicas e dispositivos percutâneos, abriram-se novas perspectivas para o tratamento do tromboembolismo pulmonar ${ }^{2}$. A intervenção percutânea proporciona tratamento rápido e menos agressivo que a trombectomia cirúrgica e, além disso, permite avaliar o local da obstrução, o tamanho do trombo e a anatomia por meio da arteriografia pulmonar. Estão sendo utilizados vários dispositivos e cateteres para retirada de trombos, com fragmentação e/ou aspiração, levando à liberação do fluxo pulmo- nar, com bons resultados iniciais; no entanto, ainda não há consenso e ensaios clínicos randomizados para embolectomia percutânea no tromboembolismo pulmonar maciço. Uma meta-análise comparou o uso de cateteres Amplatz e Greenfield e de dispositivos de fragmentação Angiojet e Hydrolyser combinado ou não com o uso de trombolítico local (intrapulmonar) no tratamento do tromboembolismo pulmonar maciçO $^{11}$. Os melhores resultados foram conseguidos com os dispositivos de fragmentação reolítica associados a trombolítico local (com Angiojet isolado o sucesso clínico foi de 75\%; e em associação com trombólise intra-arterial, o sucesso foi de $87 \%)^{12,13}$. Outro estudo, publicado recentemente, relatou a associação de cateter de fragmentação (pig tail) e sistema de aspiração (Aspirex) em 18 pacientes com tromboembolismo pulmonar maciço, com sucesso clínico em 88,8\% e complicações maiores (óbito, hemorragia cerebral e sangramentos) em $11,1 \%{ }^{14}$.

No nosso caso, a paciente apresentava idade bastante avançada e cirurgia de grande porte recente (operação no quadril há 26 dias), com alto risco de hemorragia no caso do tratamento convencional com trombólise sistêmica. Encontrava-se em choque cardiogênico refratário a drogas vasoativas (altas doses de dopamina e noradrenalina) e a intervenção percutânea rápida, para restabelecimento do fluxo pulmonar, reverteu a insuficiência cardíaca direita, com melhora hemodinâmica rápida e boa qualidade de vida. No caso relatado, usamos um cateter de aspiração a vácuo, o cateter Pronto 0,035", lançado recentemente, bastante flexível e com bom lúmen (10 F), permitindo aspirar grande quantidade de trombos. Esse cateter tem a vantagem de ser facilmente operado e é indicado para vasos com diâmetro $\geq 4 \mathrm{~mm}$, em casos agudos de tromboembolismo, idealmente nas primeiras 48 horas do evento. Conseguimos aspirar grande quantidade de trombos e a associação de trombólise intra-arterial, com dose reduzida (menor risco de sangramentos) propiciou excelente evolução clínica.

Como o uso desse cateter de aspiração para casos de tromboembolismo pulmonar é muito recente, necessitamos de maiores estudos para comprovar a eficácia e a melhora da sobrevida, para que possamos propagar mais esse tipo de tratamento para essa grave doença. Temos poucos casos relatados, algumas revisões e limitado acesso a novos dispositivos em nosso meio. Devemos lembrar que ainda não há evidências para o uso rotineiro desses cateteres e dispositivos, e que a embolectomia por meio de cateteres é indicada quando houver comprometimento de artéria pulmonar principal e dos ramos lobares (e não dos ramos segmentares $)^{15}$. O tromboembolismo pulmonar é uma condição clínica crítica e fatal, muitas vezes não diagnosticada, com grandes dificuldades no manejo, que requer tratamento rápido, e a melhora da terapêutica é necessária para diminuir a mortalidade ${ }^{16,17}$. 


\section{CONCLUSÃO}

Relatamos um caso de tromboembolismo pulmonar maciço em paciente idosa, com grande instabilidade clínica e hemodinâmica, que foi submetida a intervenção percutânea precoce. Realizamos embolectomia com cateter de aspiração e trombólise intraarterial (dose reduzida), com excelente resultado. Necessitamos de maiores estudos, com intervenção percutânea rápida, com esse tipo de cateter de fácil manejo, para tratarmos melhor essa doença de grande mortalidade.

\section{CONFLITO DE INTERESSES}

Os autores declararam inexistência de conflito de interesses.

\section{REFERÊNCIAS BIBLIOGRÁFICAS}

1. Dalen JE, Alpert JS. Natural history of pulmonary embolism. Prog Cardiovasc Dis. 1975;17(4):259-70.

2. Uflacker R. Interventional therapy for pulmonary embolism. J Vasc Interv Radiol. 2001;12(2):147-64.

3. Wood KE. Major pulmonary embolism: review of a pathophysiologic approach to the golden hour of hemodynamically significant pulmonary embolism. Chest. 2002;121(3):877-905.

4. Torbicki A, van Beek EJR, Charbonnier B, Meyer G, Morpurgo $M$, Palla $A$, et al. Guidelines on diagnosis and management of acute pulmonary embolism. Task Force Report on Pulmonary Embolism, European Society of Cardiology. Eur Heart J. 2000; 21(16):1301-36.

5. Lilienfeld DE, Chan E, Ehland J, Godbold JH, Landrigan PJ, Marsh G. Mortality from pulmonary embolism in the United States: 1962 to 1984. Chest. 1990;98(5):1067-72.

6. Elliot CG. Pulmonary physiology during pulmonary embolism. Chest. 1992;101(4 Suppl):163S-71.
7. Kucher N, Rossi E, De Rosa M, Goldhaber SZ. Massive pulmonary embolism. Circulation. 2006;113(4):577-82.

8. Meneveau N, Seronde MF, Blonde MC, Legalery P, DidierPetit K, Briand F, et al. Management of unsuccessful thrombolysis in acute massive pulmonary embolism. Chest. 2006;129(4): 1043-50.

9. Kasper W, Konstantinides S, Geibel A, Olschewski M, Heinrich F, Grosser KD, et al. Management strategies and determinants of outcome in acute major pulmonary embolism: results of a multicenter registry. J Am Coll Cardiol. 1997;30(5): 1165-71.

10. Stulz P, Schläpfer R, Feer R, Habicht J, Grädel E. Decision making in the surgical treatment of massive pulmonary embolism. Eur J Cardiothorac Surg. 1994;8(4):188-93.

11. Skaf E, Beemath A, Siddiqui $T$, Janjua $M$, Patel NR, Stein PD. Catheter-tip embolectomy in the management of acute massive pulmonary embolism. Am J Cardiol. 2007;99(3):415-20.

12. Zeni Jr PT, Blank BG, Peeler DW. Use of rheolytic thrombectomy in treatment of acute massive pulmonary embolism. J Vasc Interv Radiol. 2003;14(12):1511-5.

13. Siablis D, Karnabatidis D, Katsanos K, Kagadis GC, Zabakis P, Hahalis G. Angiojet rheolytic thrombectomy versus local intrapulmonary thrombolysis in massive pulmonary embolism: a retrospective data analysis. J Endovasc Ther. 2005; 12(2):206-14.

14. Eid-Lidt G, Gaspar J, Sandoval J, Santos FD, Pulido T, González Pacheco $\mathrm{H}$, et al. Combined clot fragmentation and aspiration in patients with acute pulmonary embolism. Chest. 2008;134(1):54-60.

15. Kucher N, Goldhaber SZ. Management of massive pulmonary embolism. Circulation. 2005;112(2):e28-32.

16. Suarez JA, Meyerrose GE, Phisitkul S, Kennedy S, Roongsritong C, Tsikouris J, et al. Review of catheter thrombectomy devices. Cardiology. 2004;102(1):11-5.

17. Brahmbhatt T, Tutton S, Mannebach P, Cinquegrani M. Rheolytic thrombectomy in patient with massive pulmonary embolism: a case report and review of literature. Catheter Cardiovasc Interv. 2007;70(6):802-6. 